\title{
Clinical significance of blood-based miRNAs as biomarkers of non-small cell lung cancer (Review)
}

\author{
LIN LI, YU SUN, MIN FENG, LIANG WANG and JING LIU \\ Stem Cell Clinical Research Center, National Joint Engineering Laboratory, Regenerative Medicine Centre, \\ First Affiliated Hospital of Dalian Medical University, Dalian, Liaoning 116011, P.R. China
}

Received June 20, 2017; Accepted January 5, 2018

DOI: $10.3892 / \mathrm{ol} .2018 .8469$

\begin{abstract}
Non-small-cell lung cancer (NSCLC) accounts for $85 \%$ of all cases of lung cancer. However, the predicted 5-year survival rate of patients with NSCLC is only $15.9 \%$. microRNAs (miRNAs) are single-stranded, noncoding RNA molecules that are easily detectable in blood in a non-invasive manner, with features of stability, reproducibility and consistency in blood. Therefore, miRNAs derived from blood are able to have a significant impact on NSCLC diagnosis, metastasis and targeted therapies. Compared with the clinical protein markers carcinoembryonic antigen, cytokeratin fragment 21-1 and cancer antigen-125, blood-based miRNAs also display a higher diagnostic efficacy in NSCLC. Exosomal miRNAs are identified to be easily measured and have the potential to be used as diagnostic biomarkers in NSCLC, therefore providing an alternative method of biopsy profiling. The miRNA profile in exosomes is similar to the profile in primary tumor, meaning that this feature may be a powerful tool for NSCLC clinical diagnosis and targeted therapies. The focus of the present review was the clinical significance of blood-based exosomal miRNAs in diagnosis, prognosis, metastasis and targeted therapies of NSCLC.
\end{abstract}

\section{Contents}

1. Introduction

2. Clinical significance of miRNAs derived from blood of NSCLC

3. Clinical significance of exosomal miRNAs derived from the blood of patients with NSCLC

Correspondence to: Professor Jing Liu, Stem Cell Clinical Research Center, National Joint Engineering Laboratory, Regenerative Medicine Centre, First Affiliated Hospital of Dalian Medical University, 222 Zhongshan Road, Dalian, Liaoning 116011, P.R. China

E-mail: liujing@dmu.edu.cn

Key words: microRNAs, non-small cell lung cancer, biomarker, exosomes, diagnosis, metastasis, targeted therapies
4. Clinical significance of miRNAs derived from tissue of NSCLC

5. Conclusion

\section{Introduction}

The mortality rate of lung cancer has increased 6.9-fold in China from 1973 to 2014 (1). In 2015, there were 4,292,000 new cancer cases and 2,814,000 incidences of cancer-associated mortality in China, with lung cancer the principal cause of this mortality (2). Non-small cell lung cancer (NSCLC) accounts for $\sim 85 \%$ of all cases of lung cancer (3). NSCLC is a heterogeneous disease comprised of common cancer subtypes, including squamous cell carcinoma (SCC), adenocarcinoma and large cell carcinoma. Although novel therapeutics have alleviated the disease burden and enhanced the overall quality of life in patients with NSCLC, the 5-year survival rate of patients with NSCLC remains low, at $15.9 \%$ (4). The poor rate of early detection and limited efficacy of presently available therapies for advanced-stage NSCLC are the cause of the low 5-year survival rate of patients with NSCLC (5). Current diagnostic methods for NSCLC include chest radiography, sputum cytology, computed tomography (CT) or assessment of a combination of clinical protein markers of tumors. These markers include carcinoembryonic antigen (CEA), neuron-specific enolase, cytokeratin fragment 21-1 and tissue polypeptide-specific antigen; these protein markers have previously been evaluated in large-scale clinical trials (1-3). However, these current diagnostic and prognostic methods have a number of undesirable side effects, Examples of these side effects include the high erroneous diagnostic rate, the low sensitivity and specificity (5).

MicroRNAs (miRNAs/miRs) represent an appealing alternative for assessing the diagnosis and prognosis of NSCLC as they can be detected in the blood in a non-invasive manner. miRNAs are endogenous, noncoding RNA molecules $\sim 20$ nucleotides in length that have a marked influence on the biological functions of single cells and complete organisms (6). A number of prior studies have validated that miRNAs can be readily detected in the blood, and that miRNAs are released by three different mechanisms: Energy-free passive leakage from lysed cells, active release through microvesicles and active secretion in the microvesicles free form. For example, 
serum miRNAs remain stable under harsh conditions, including boiling, acidic ( $\mathrm{pH}$ 1.0) or alkaline ( $\mathrm{pH}$ 13.0) solutions, long-term storage and undergoing multiple freeze-thaw cycles. Furthermore, serum miRNAs in healthy subjects are also consistent, with a Pearson correlation coefficient close to 1 (6). Therefore, the present review focuses on miRNAs derived from blood samples.

There are three principal membrane vesicle types: i) Microvesicles, ii) exosome vesicles, and iii) apoptotic vesicles (7). Microvesicles, also termed shedding vesicles, are large (>200 nm diameter), dense, and include phosphatidylserine, integrins and CD40 ligand (8). Exosomes are spherical nano-sized vesicles, with a round cup-shaped morphology, a density of 1.13-1.19 g/ml and a diameter of 40-100 nm (9). Exosomes contain large amounts of molecular cargo, including mRNAs, miRNAs, proteins and DNA, which are protected by a lipid bilayer. In 2007, Valadi et al (10) identified exosomal miRNAs, which were subsequently identified to be obtained readily, had the potential to be used as diagnostic markers and could serve as an alternative for biopsy profiling. They are suitable for profiling as the miRNA profile in exosomes exhibit similarities to primary tumor profile (11); therefore, this feature may be a powerful tool for early diagnosis, prognosis, metastasis and targeted therapy in NSCLC.

\section{Clinical significance of miRNAs derived from blood of NSCLC}

Blood-based miRNAs and clinical diagnosis of NSCLC. According to clinical analysis of stage I to IV cases of NSCLC, the mean risk score and high risk-score rate progressively increased as the stage increased (Table I). Conversely, the false-positive rate progressively decreased (3). The Tumor-Node-Metastasis (TNM) stage (12) of patients with NSCLC was also associated with the expression level of blood-based miRNAs. A previous study demonstrated that twelve plasma miRNAs could significantly discriminate between stages I-III of patients with NSCLC and controls (13). Levels of blood-based miRNAs could also be used to distinguish early stages (stages I and II) from advanced stages (stages III and IV) of NSCLC. In the present review, blood-based miRNAs are divided into three categories: Serum-based miRNAs, plasma-based miRNAs and miRNAs derived from peripheral blood mononuclear cells (PBMCs).

Blood-based miRNAs and early stage of NSCLC. Early detection is particularly crucial for the initial stage diagnosis of patients with NSCLC who present without clinical symptoms. The properties of blood-based miRNAs could predict disease probability irrespective of whether the patient is asymptomatic or symptomatic, and also is able to distinguish benign from malignant lesions. Furthermore, miRNAs could determine the onset of the malignant disease in individual patients over time (14-16). Serum-based miRNAs had been identified differentially between patients with early-stage NSCLC and controls: hsa-miR-1254 and hsa-miR-574-5p were evidently increased in early-stage NSCLC samples (compared with the control group) in the identification and validation cohort of the study by Foss et al (14). The combination of miR-125a-5p, miR-25 and miR-126 could also discriminate between NSCLC

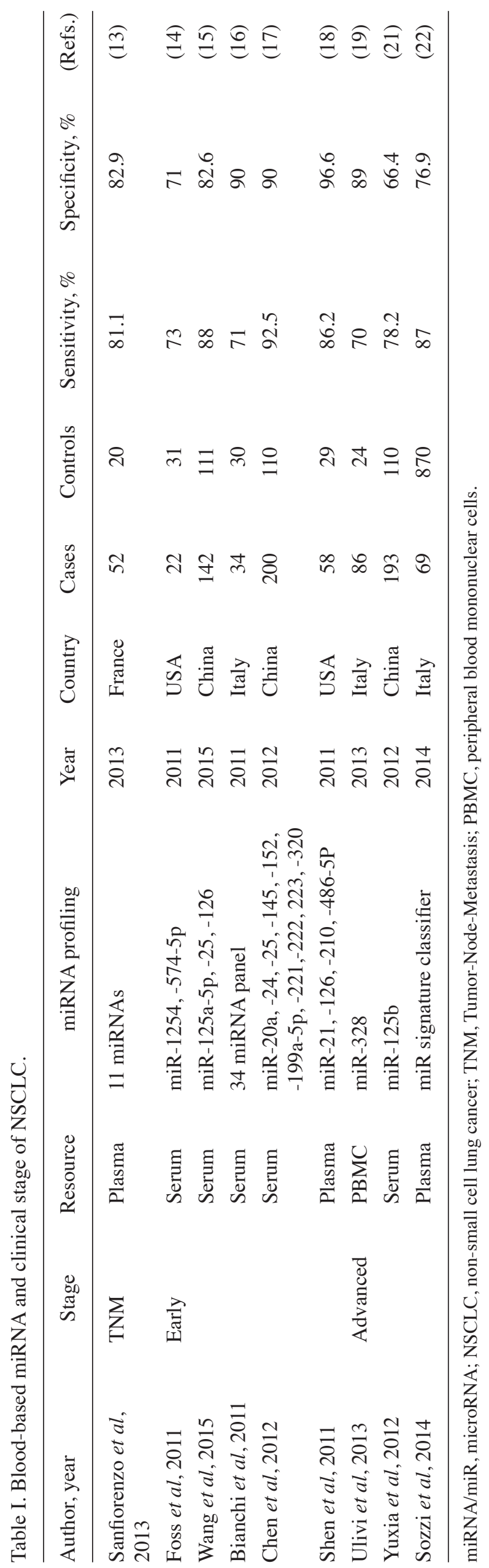


patients and controls (15). Bianchi et al detected 34 miRNAs in the serum that were able to distinguish patients with early stage NSCLCs from asymptomatic high-risk individuals with $80 \%$ accuracy (16). In a retrospective study, 10-serum miRNA for the early detection of NSCLC could precisely classify serum samples which were collected 33 months ago (17). Similarly, the four miRNAs (miRNA-21, -126, -210, and 486-5p) derived from plasma were also identified as stable and reliable in discriminating patients with NSCLC from controls (18). Furthermore, using miRNAs also produced high sensitivities and specificities in validating the presence of stage I NSCLC. A further study revealed that miR-328 derived from the PBMCs was the most effective diagnostic discriminator in the group of early stage tumors (19).

Blood-based miRNAs and advanced stage of NSCLC. Patients with stage III/IV NSCLC usually have a poor prognosis: The majority of these patients have a median survival of $\sim 1$ year (20). Clinicopathological criteria, including age, histological type, TNM stage and treatment method, are frequently used prediction factors for the prognosis of NSCLC. Serum miR-125b was markedly associated with poor prognosis in NSCLC in a prior study (21). A study concerning a large number of plasma samples indicated that the miRNAs signature classifier possessed predictive, diagnostic and prognostic significance, and could reduce the false-positive rate of low-dose CT (22). Low miR-10b expression in patients with stage I-II carcinoma was a positive indicator for the clinical prognosis of NSCLC, whereas high miRNA-10b expression in stage III-IV carcinoma was a negative indicator of the clinical prognosis of NSCLC. Furthermore, the 5-year survival rate of the low miR-10b expression group was markedly higher than the high miR-10b expression group (23).

Blood-based miRNAs and clinical protein markers CEA, Cyfra21-1 and CA125 in NSCLC. Recently, serum-based miRNAs were identified to have a higher diagnostic efficacy than the clinical protein markers CEA, CA125 and Cyfra21-1 in patients with NSCLC when compared with controls. A large number of samples were used to validate the authenticity of the results obtained and reproducibility of the experiments. A total of 70 patients with stage I NSCLC and 48 controls were collected in order to analyze the expression levels of miR-29c, miR-93 and miR-429 with CEA. miR-29c, miR-429 and CEA had an area under the curve (AUC; 0.833) higher than single serum miR-29c (0.727) and miR-429 (0.723), all of which were higher than CEA (0.534) (24). Serum specimens from 112 patients with NSCLC and 104 controls were subjected to research the levels of miR-182, miR-183, miR-126 and miR-210. These four miRNAs in conjunction with CEA were further validated by the AUC of 0.965 , with a high sensitivity (88.5\%) and specificity (92.5\%) (25). Zhou et al (26) screened 396 serum samples from 252 patients with NSCLC and 144 healthy individuals, finding that miR-652 together with miR-660 had a markedly higher diagnostic value than CEA and CA125 for distinguishing between patients with NSCLC/adenocarcinoma from controls in the training and test cohort. The miR652 ${ }^{+}$miR- $660^{+}$Cyfra21-1 model had the highest clinical value for distinguishing patients with NSCLC from controls, which displayed higher early diagnostic value compared with the clinical value of the double miRNA models and Cyfra21-1 alone, in the training and test cohort (26). Table II surmises the findings of studies with respect to blood-based miRNAs and clinical protein markers CEA, Cyfra21-1 and CA125.

Blood-based miRNAs and metastasis of NSCLC. miRNA expression levels may be associated with distant metastasis, prognosis and TNM stage of the tumor. Metastasis is the principal cause of mortality in patients with tumors (27). A large number of miRNAs had been demonstrated to be involved in cancer metastasis. For example, studies had demonstrated that miR155, miR-222 and miR-107 serve a role in pancreatic cancer $(27,28)$. miR-200 was involved in gastric cancer (29) and miR-133b acted in colorectal cancer (30). Therefore, an improved understanding of the role of miRNA expression in the metastasis of NSCLC may result in an improved understanding of disease development and an improvement in the detection and therapy of NSCLC.

Metastasis is a multistep procedure, in which, tumor cells lose their adhesion to the stroma, pass through the basal membrane, then move into the blood vessels, alive in the blood circulation, attached to the blood stream, extravasate and proliferation in the host organ. Tumor cells either enter a dormant state or proliferate a form that shaping from multicellular epithelial-mesenchymal transition (EMT) of NSCLC cells (31-33). miR-152 regulated the proliferation and invasion of NSCLC cells by downregulating basic fibroblast growth factor (FGF2) (34). miR-194 suppressed the proliferation, migration, invasion and metastasis by EMT of NSCLC cells that form lung metastases in vivo (35). miRNA-449a induced $\mathrm{G}_{1}$ arrest, apoptosis and cellular senescence (36). An in vitro study demonstrated that miR-449 inhibited cell migration and invasion in NSCLC, in part by targeting c-Met (36). A total of 74 patients with NSCLC were selected for two groups (high and low expression of miRNA10b), and the low expression of miRNA10b, metastasis, with stage III-IV carcinoma was identified to indicate poor prognosis in patients with NSCLC (22).

Blood-based miRNAs and targeted therapies of NSCLC. Chemotherapy and molecular targeted therapies are generally applied either alone or in conjunction with surgery and radiotherapy to treat patients with NSCLC (Table III) (37). miRNAs may act as molecular tumor biomarkers, predicting the incidence of multidrug resistance in NSCLC. Epidermal growth factor receptor (EGFR)-mutated or anaplastic lymphoma kinase (ALK)-rearranged tumors of patients with NSCLC could be treated with tyrosine kinase inhibitors (TKIs) including, erlotinib, gefitinib, crizotinib and ceritinib $(37,38)$. For instance, one mechanism of action of erlotinib was the regulation of miR-9-Foxo1 in NSCLC (39). It is hypothesized that miR-21 could maintain the acquired resistance of EGFR-TKI in NSCLC by downregulation of phosphatase and tensin homolog and programmed cell death 4 , and activation of the phosphoinositide 3-kinase/protein kinase B pathway (40). miR-20a regulated expression of the iron exporter ferroportin in NSCLC (41). miR-512-5p and miR-373 expression increased cisplatin-induced apoptosis in lung cancer and the re-expression of miRNAs did benefit 


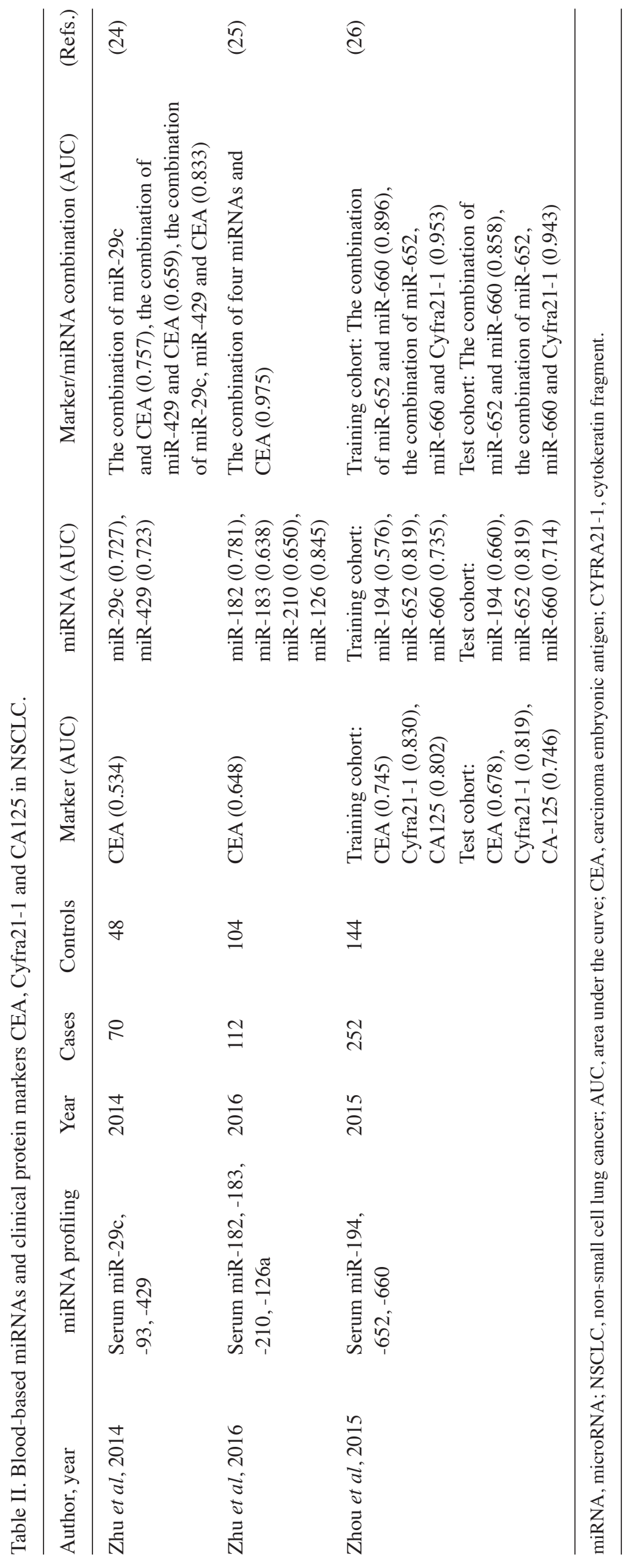




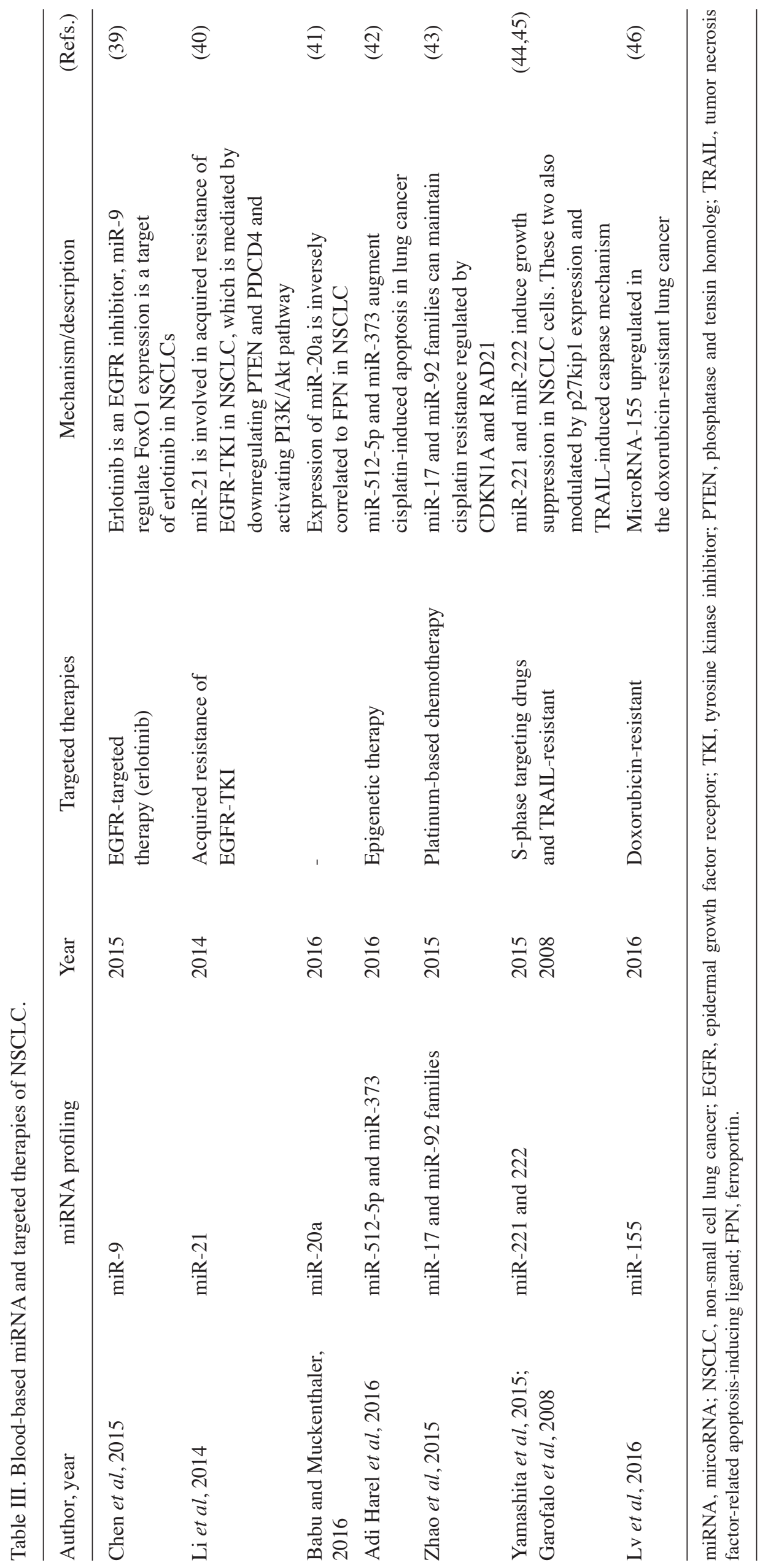


from epigenetic cancer therapy (42). The miR-17 and miR-92 families were involved in cisplatin resistance and were regulated by cyclin-dependent kinase $1 \mathrm{~A}$ and double-strand-break repair protein RAD21 homolog in NSCLC. These major factors contributed to cisplatin resistance, potentially by suppressing DNA synthesis and the repair of DNA damage (43). miR-221 and miR-222 increased the chemosensitivity to S-phase-targeting drugs, cisplatin and gemcitabine by inducing growth suppression in NSCLC cells (44). These two miRNAs were also regulated by p27kip1 and tumor necrosis factor-related apoptosis-inducing ligand-induced caspase mechanism (45). A previous study demonstrated that miRNA-155 was upregulated in the doxorubicin-resistant lung cancer A549/dox cell line (46).

\section{Clinical significance of exosomal miRNAs derived from the blood of patients with NSCLC}

Blood-based exosomal miRNAs and clinical diagnosis of NSCLC. Tumor cells produce an increased number of exosomes compared with healthy organs and cells (47). There are approximately $>3$ billion exosomes $/ \mathrm{ml}$ in the blood of cancer patients, which is almost twice the concentration than in the blood of healthy controls (48). Exosomal miRNAs are similar to the miRNAs of parental cancer cells, resulting in an increased amount of research into exosomal miRNAs for cancer diagnosis. A number of examples of potential clinical applications for identification of high levels of exosomal miRNAs have been identified in melanoma, glioblastoma, esophageal squamous cell carcinoma, pancreatic and prostate cancer (Table IV) (47-50).

Blood-based exosomal miRNAs and early stage of NSCLC. A number of studies have previously attempted to validate that exosomal miRNAs may be used as a biomarker for the detection, diagnosis and metastatic spread of cancer. A study involving 27 patients with lung adenocarcinoma and 9 controls were selected to validate the special 12 miRNAs; the presence of the 12 miRNAs were also mirrored in the circulating exosomes (51). Four miRNAs (miR-378a, miR-379, miR-139-5p and miR-200b-5p) were enrolled for screening tests and six miRNAs (miR-151a-5p, miR-30a-3p, miR-200b-5p, miR-629, miR-100 and miR-154-3p) were enrolled for diagnostic tests, all of which have demonstrated high sensitivities and specificities of patients with lung adenocarcinomas and controls (52). A prospective analysis by Rodriguez et al (53) regarding blood and bronchoalveolar lavage (BAL) samples derived from 30 patients with NSCLC and 75 controls demonstrated that exosomes and miRNAs levels were higher in the plasma and BAL from patients with NSCLC. Zhou et al (54) identified six upregulated plasma miRNAs (miR-19b3p, miR-21-5p, miR-221-3p, miR-409-3p, miR-425-5p and miR-584-5p). These miRNAs were able to distinguish between patients with lung adenocarcinoma and healthy controls, with a receiver operating characteristic curve (ROC) of $0.72,0.74$ and 0.84 for the training, testing and the external validation stage, respectively. Additionally, levels of miR-19-3p, miR-21-5p and miR-221-3p were significantly upregulated in exosomes derived from peripheral plasma samples of patients with lung adenocarcinoma (54).
Blood-based exosomal miRNAs and advanced stage of NSCLC. The vesicle-associated miRNAs let-7f and miR30e-3p could be used to discriminate between two groups of patients for different tumor stages and therefore the surgical options available to the patients. Notably, the two miRNAs examined in NSCLC have also been associated with a poor clinical outcome (55). A follow-up survey revealed that the levels of miR-205, miR-19a, miR-19b, miR-30b and miR-20a from the plasma of patients was evidently decreased following SCC surgery (resection). Assessment of miRNA levels in patients with lung SCC revealed the presence of high levels of exosomal miRNAs expression in tumor (56). The presence of exosomal miR-23b-3p, miR-10b-5p and miR-21-5p were identified as prognostic biomarkers for patients with NSCLC. The median follow-up time was 14.40 months (range, 3.43-36.87 months), with $71(36.22 \%)$ patients succumbing to disease by the end of the experiment (57). These data indicate that the novel biomarkers and the technology for the detection of exosomal miRNAs for NSCLC are vital to enhance the sensitivity and specificity, which may be essential for improving the overall survival of patients.

Blood-based exosomal miRNAs and metastasis of NSCLC. (Fig. 1) Extracellular vehicles (EVs) deliver nucleic acids to target cells, allowing for the exchange of genetic information between cells. EVs are also capable of altering the phenotype of neighboring cells (58). Therefore, exosomes can not only promote the biological processes of tumors, but also influence the metastatic signatures of malignant tumors. Additionally, the regulatory signatures of tumor-derived exosomes are important for shaping the tumor microenvironment (31).

Exosomes, as mediators of EMT, are involved in the migration and invasion of metastasis (31). EMT continually initiates the process of metastasis, where tumor cells lose their polarity and cell-cell junctions and acquire migratory and invasive capabilities with a low proliferation state (32). If the tumor cells reach the distant pre-metastatic niche, the reverse process occurs (33). Moreover, when EMT cells arrive at the metastatic side, these EMT cells undergo epithelial to mesenchymal transition. (31). For example, exosomal miR-23a sustained the EMT-promoting effect of transforming growth factor- $\beta 1$ by suppressing E-cadherin synthesis in lung carcinoma $(59,60)$. Tumor-derived exosomes can lead cancer cells to acquire a mesenchymal phenotype and can convert mesenchymal stem cells into cancer-associated fibroblasts following an increase in the expression of $\alpha$-smooth muscle actin (61). Exosome-derived miR-302b was also demonstrated to inhibit cell proliferation and migration in lung cancer (62).

Exosomal blood-based miRNAs and targeted therapies of NSCLC. As in artificial loaders of signaling molecules, exosomes possess signatures, including biocompatibility, stability, biological barrier permeability, low immunogenicity and low toxicity (58). These features make exosomes attractive for therapeutic use. Additionally, exosomes are able to escape rapid clearance by the mononuclear phagocyte system owing to their small size (63). Xiao et al (64) reported that A549 cell-derived exosomes reduced the sensitivity of non-treated A549 cells to cisplatin, following cisplatin exposure. Notably, the phospholipid constituents of EV were markedly 


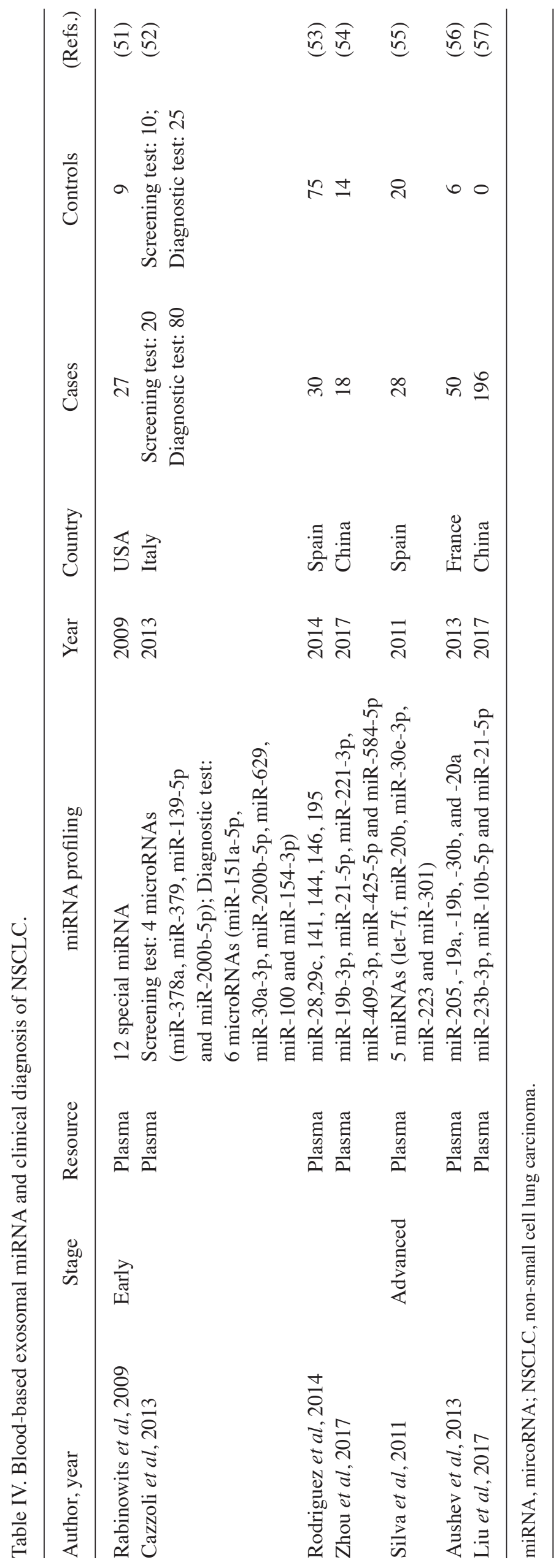




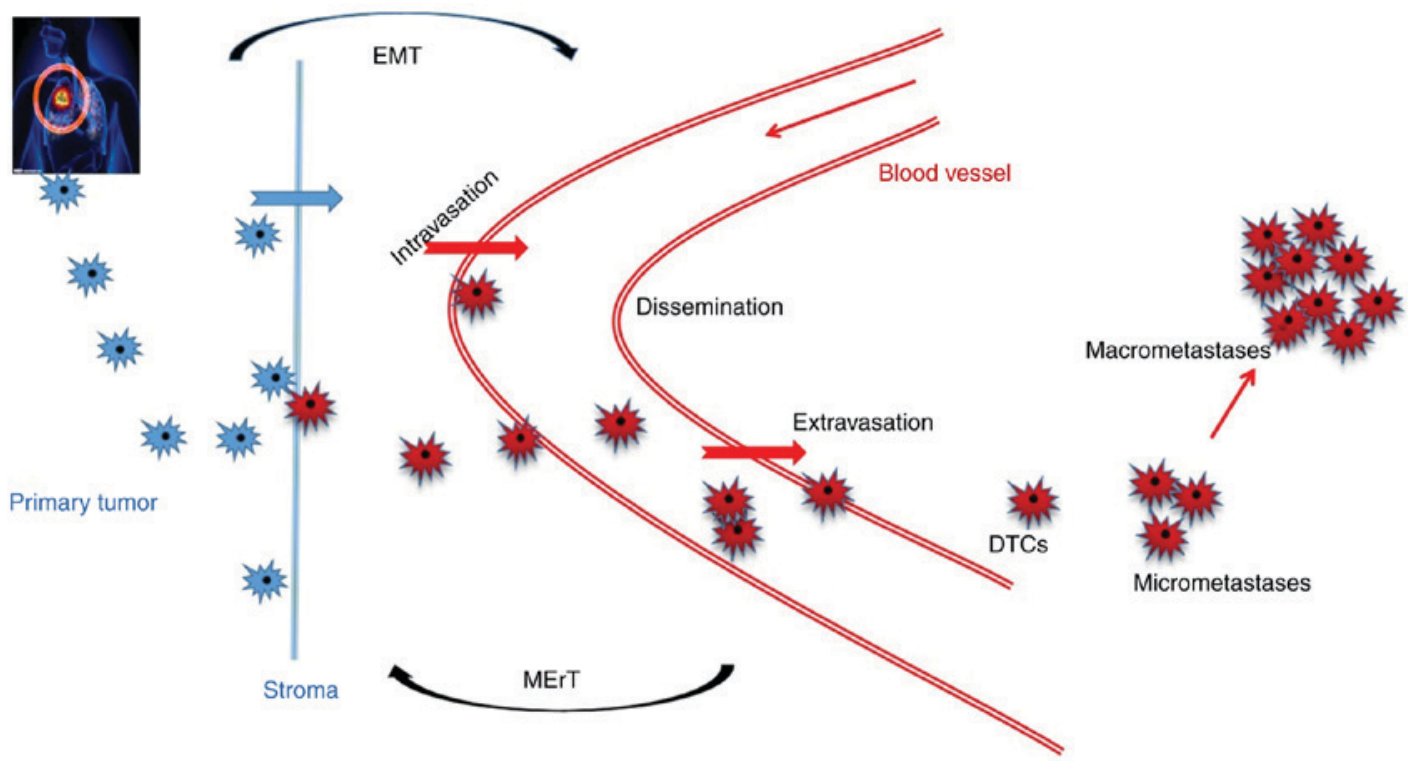

Figure 1. Metastasis is a multistep procedure, in which tumor cells lose their adhesion to the stroma, pass through the basal membrane, then move into blood vessels and enter blood circulation where they then extravasate and proliferate in the host organ. Moreover, EMT cells arriving at the metastatic side need to undergo a MET. Exosomes, as mediators of EMT, are involved in the migration and invasion of metastasis. EMT continually initiates the process of metastasis. This is where tumor cells lose their polarity and cell-cell junctions, and acquire migratory and invasive capabilities with a low proliferative rate. If the tumor cells reach the distant pre-metastatic niche, the reverse process occurs.

distinguished in gefitinib-resistant NSCLC. Sophisticated mass-spectrometry-based shotgun lipidomic assays were performed for in-depth analysis. Lipid matrix-assisted laser desorption/ionization analysis revealed that EV phospholipid composition was significantly more distinct in PC9R cells compared with PC9 cells (65). There are three techniques to load an exosome with a therapeutic miRNA mimic or antagonist: Co-transfection with two plasmids, or co-transduction with two viruses; electroporation; or the transient transfection of miRNAs $(66,67)$.

Several trials have been conducted to assess the safety of EV-based antitumor and antibacterial vaccines. This included a phase I trial that administered EVs from autologous DCs pulsed with melanoma antigen gene peptides to patients with NSCLC (68). Furthermore, a previous phase II trial was also conducted in order to evaluate interferon- $\gamma$-dendritic cell-derived exosomes loaded with major histocompatibility complex I/II-restricted cancer antigens following chemotherapeutic induction in patients with inoperable NSCLC without tumor progression (69). These results indicate that DC-derived exosomes vaccines may be safe and may promote T-cell and natural killer cell responses in patients. The extracorporeal hemofiltration of circulating EVs is a novel potential strategy, and has been previously proposed to be a therapeutic strategy for patients with cancer $(70,71)$.

\section{Clinical significance of miRNAs derived from tissue of NSCLC}

In addition to blood samples, studies have demonstrated that miRNAs are also obtained from a large number of tissue specimens of patients with NSCLC and controls to validate the potential clinical significance of miRNAs in NSCLC. For example, miR-30d-5p was demonstrated to be downregulated in NSCLC tissues, with a 2-fold difference in expression between tumor tissues and the corresponding para-tumorous tissues (72). In 2006 a study describing the diagnostic miRNA signatures of NSCLC revealed that 12 specific miRNAs were overexpressed when compared with normal lung tissue (73). miRNAs extracted from tissues have been identified to be a more direct and intuitive method; however, tissue as another sample resource, are not the primary focus of the present review.

\section{Conclusion}

Currently, circulating tumor cells, circulating tumor DNA and exosomes, are all covered in the concept 'liquid biopsies'. A liquid biopsy is a liquid biomarker that may be easily isolated from numerous body fluids (blood, saliva, urine, ascites and pleural effusion and a tissue biopsy, a representative of the tissue from which it is obtained (74). Exosomes, as a non-invasive means of performing 'liquid biopsies', may be used for early diagnosis, obtaining prognostic information, metastasis development, real-time monitoring of tumor stages and understanding of therapeutic targets in patients with NSCLC.

The present review focuses on the clinical significance of blood-based exosomal miRNAs in the detection, metastasis and therapies of NSCLC. As a novel means of intercellular communication, exosomes can stimulate the growth, invasiveness and metastasis of NSCLC (75). Compared with the conventional methods used for clinical diagnosis of NSCLC, exosomes have numerous advantages: Exosomes contain an increased amount of genetic information, which can spread widely throughout the bodily fluids; exosomes have a relatively long circulating half-life with drugs in vivo; exosomes secreted by cells exhibit target selection; exosomes can enhance their cell-specific targeting function by altering their membrane; and exosomes can carry drugs in vivo and in vitro (76,77). According to recent findings in NSCLC, the significant difference in miRNAs and exosomal miRNAs derived from blood between patients with 
NSCLC and controls, and the similarities between exosomal miRNAs and miRNAs all indicate that exosomal miRNAs may be useful as a biomarker for clinical diagnosis in NSCLC (76).

A number of problems surrounding the use of exosomes require addressing, which include: A lack of a reliable and cost-effective detection platforms for exosomal miRNAs in routine clinical settings; the complication of procedures, including specificity screening, detection methods suitable for the selection of the reference genes; the apparent shortcomings, which include time-consuming experiments, expensive instruments and a heavy reliance on the sample-handling skills; and a lack of research into the mechanism of action of exosomes.

Although research into exosomal miRNAs and NSCLC in its infancy, with the development of detection methods and the maturity of the research, in the future blood-based exosomal miRNAs may have prospects for application in terms of prediction, diagnosis, metastasis, prognostic evaluation and individualized treatment in lung cancer. Therefore, identifying novel therapeutic strategies and acquiring a better understanding of the biological functions of exosomal miRNAs as biomarkers in NSCLC will be of the utmost relevance in modern molecular oncology.

\section{Acknowledgements}

Not applicable.

\section{Funding}

The presents study was supported by the National Nature Science Foundation of China (grant no. 81471308).

\section{Availability of data and materials}

The datasets generated and analysed in the present study are included in this published article.

\section{Authors' contributions}

JL devised the study topic. YS and LW revised the paper. MF provided additional clinical knowledge. LL wrote this manuscript.

\section{Ethics and consent to participate}

Not applicable.

\section{Consent for publication}

Not applicable.

\section{Competing interests}

The authors declare that they have no competing interests.

\section{References}

1. Shen X, Wang L and Zhu L: Spatial analysis of regional factors and lung cancer mortality in China, 1973-2013. Cancer Epidemiol Biomarkers Prev 26: 569-577, 2017.
2. Chen W, Zheng R, Baade PD, Zhang S, Zeng H, Bray F, Jemal A, Yu XQ and He J: Cancer statistics in China, 2015. CA Cancer J Clin 66: 115-132, 2016.

3. Fujita Y, Kosaka N, Araya J, Kuwano K and Ochiya T: Extracellular vesicles in lung microenvironment and pathogenesis. Trends Mol Med 21: 533-542, 2015.

4. Chen Z, Fillmore CM, Hammerman PS, Kim CF and Wong KK: Non-small-cell lung cancers: A heterogeneous set of diseases. Nat Rev Cancer 14: 535-546, 2014.

5. Jemal A, Siegel R, Ward E, Hao Y, Xu J and Thun MJ: Cancer statistics, 2009. CA Cancer J Clin 59: 225-249, 2009.

6. Chen X, Ba Y, Ma L, Cai X, Yin Y, Wang K, Guo J, Zhang Y, Chen J, Guo X, et al: Characterization of microRNAs in serum: A novel class of biomarkers for diagnosis of cancer and other diseases. Cell Res 18: 997-1006, 2008.

7. Théry C, Ostrowski M and Segura E: Membrane vesicles as conveyors of immune responses. Nat Rev Immunol 9: 581-593, 2009.

8. Cocucci E, Racchetti G and Meldolesi J: Shedding microvesicles: Artefacts no more. Trends Cell Biol 19: 43-51, 2009.

9. Simons M and Raposo G: Exosomes-vesicular carriers for intercellular communication. Curr Opin Cell Biol 21: 575-581, 2009.

10. Valadi H,Ekström K, Bossios A, Sjöstrand M, Lee JJ and Lötvall JO: Exosome-mediated transfer of mRNAs and microRNAs is a novel mechanism of genetic exchange between cells. Nat Cell Biol 9: 654-659, 2007.

11. Jaiswal R, Gong J, Sambasivam S, Combes V, Mathys JM, Davey R, Grau GE and Bebawy M: Microparticle-associated nucleic acids mediate trait dominance in cancer. FASEB J 26: 420-429, 2012.

12. Edge SB and Compton CC: The American Joint Committee on Cancer: The 7th edition of the AJCC cancer staging manual and the future of TNM. Ann Surg Oncol 17: 1471-1474, 2010.

13. Sanfiorenzo C, Ilie MI, Belaid A, Barlési F, Mouroux J, Marquette $\mathrm{CH}$, Brest $\mathrm{P}$ and Hofman P: Two panels of plasma microRNAs as non-invasive biomarkers for prediction of recurrence in resectable NSCLC. PLoS One 8: e54596, 2013.

14. Foss KM, Sima C, Ugolini D, Neri M, Allen KE and Weiss GJ: miR-1254 and miR-574-5p: Serum-based microRNA biomarkers for early-stage non-small cell lung cancer. J Thorac Oncol 6: 482-488, 2011.

15. Wang P, Yang D, Zhang H, Wei X, Ma T, Cheng Z, Hong Q, Hu J, Zhuo H, Song Y, et al: Early detection of lung cancer in serum by a panel of MicroRNA biomarkers. Clin Lung Cancer 16: 313-319.e1, 2015.

16. Bianchi F, Nicassio F, Marzi M, Belloni E, Dall'olio V, Bernard L, Pelosi G, Maisonneuve P, Veronesi G and Di Fiore PP: A serum circulating miRNA diagnostic test to identify asymptomatic high-risk individuals with early stage lung cancer. EMBO Mol Med 3: 495-503, 2011.

17. Chen X, Hu Z, Wang W, Ba Y, Ma L, Zhang C, Wang C, Ren Z, Zhao $\mathrm{Y}, \mathrm{Wu}$ S, et al: Identification of ten serum microRNAs from a genome-wide serum microRNA expression profile as novel noninvasive biomarkers for nonsmall cell lung cancer diagnosis. Int J Cancer 130: 1620-1628, 2012.

18. Shen J, Todd NW, Zhang H, Yu L, Lingxiao X, Mei Y, Guarnera M, Liao J, Chou A, Lu CL, et al: Plasma microRNAs as potential biomarkers for non-small-cell lung cancer. Lab Invest 91: 579-587, 2011.

19. Ulivi P, Foschi G, Mengozzi M, Scarpi E, Silvestrini R, Amadori D and Zoli W: Peripheral blood miR-328 expression as a potential biomarker for the early diagnosis of NSCLC. Int J Mol Sci 14: 10332-10342, 2013.

20. Scagliotti G, Hanna N, Fossella F, Sugarman K, Blatter J, Peterson P, Simms L and Shepherd FA: The differential efficacy of pemetrexed according to NSCLC histology: A review of two Phase III studies. Oncologist 14: 253-263, 2009.

21. Yuxia M,Zhennan T and Wei Z: Circulating miR-125b is a novel biomarker for screening non-small-cell lung cancer and predicts poor prognosis. J Cancer Res Clin Oncol 138: 2045-2050, 2012.

22. Sozzi G, Boeri M, Rossi M, Verri C, Suatoni P, Bravi F, Roz L, Conte D, Grassi M, Sverzellati N, et al: Clinical utility of a plasma-based miRNA signature classifier within computed tomography lung cancer screening: A correlative MILD trial study. J Clin Oncol 32: 768-773, 2014

23. Yang YL, Xu LP, Zhuo FL and Wang TY: Prognostic value of microRNA-10b overexpression in peripheral blood mononuclear cells of nonsmall-cell lung cancer patients. Tumour Biol 36: 7069-7075, 2015

24. Zhu W, He J, Chen D, Zhang B, Xu L, Ma H, Liu X, Zhang Y and Le H: Expression of miR-29c, miR-93 and miR-429 as potential biomarkers for detection of early stage non-small lung cancer. PLoS One 9: e87780, 2014. 
25. Zhu W, Zhou K, Zha Y, Chen D, He J, Ma H, Liu X, Le H and Zhang Y: Diagnostic value of serum miR-182, miR-183, miR-210, and miR-126 levels in patients with early-stage non-small cell lung cancer. PLoS One 11: e0153046, 2016.

26. Zhou C, Chen Z, Dong J, Li J, Shi X, Sun N, Luo M, Zhou F, Tan F and He J: Combination of serum miRNAs with Cyfra21-1 for the diagnosis of non-small cell lung cancer. Cancer Lett 367: $138-146,2015$.

27. Greither T, Grochola LF, Udelnow A, Lautenschläger C, Würl P and Taubert H: Elevated expression of microRNAs 155, 203, 210 and 222 in pancreatic tumors is associated with poorer survival. Int J Cancer 126: 73-80, 2010.

28. Lee KH, Lotterman C, Karikari C, Omura N, Feldmann G, Habbe N, Goggins MG, Mendell JT and Maitra A: Epigenetic silencing of MicroRNA miR-107 regulates cyclin-dependent kinase 6 expression in pancreatic cancer. Pancreatology 9: 293-301, 2009.

29. Ahn SM, Cha JY, Kim J, Kim D, Trang HT, Kim YM, Cho YH, Park D and Hong S: Smad3 regulates E-cadherin via miRNA-200 pathway. Oncogene 31: 3051-3059, 2012.

30. DuanFT,QianF,FangK,LinKY,WangWTandChen YQ:miR-133b, a muscle-specific microRNA, is a novel prognostic marker that participates in the progression of human colorectal cancer via regulation of CXCR4 expression. Mol Cancer 12: 164, 2013.

31. Diepenbruck M and Christofori G: Epithelial-mesenchymal transition (EMT) and metastasis: Yes, no, maybe? Curr Opin Cell Biol 43: 7-13, 2016.

32. Steinbichler TB, Dudás J, Riechelmann H and Skvortsova II: The role of exosomes in cancer metastasis. Semin Cancer Biol 44: 170-181, 2017.

33. Li J, Tan Q, Yan M, Liu L, Lin H, Zhao F, Bao G, Kong H, Ge C, Zhang F, et al: miRNA-200c inhibits invasion and metastasis of human non-small cell lung cancer by directly targeting ubiquitin specific peptidase 25. Mol Cancer 13: 166, 2014.

34. Cheng Z, Ma R, Tan W and Zhang L: MiR-152 suppresses the proliferation and invasion of NSCLC cells by inhibiting FGF2 Exp Mol Med 46: e112, 2014

35. Zhu X, Li D, Yu F, Jia C, Xie J, Ma Y, Fan S, Cai H, Luo Q, Lv Z and Fan L: miR-194 inhibits the proliferation, invasion, migration, and enhances the chemosensitivity of non-small cell lung cancer cells by targeting forkhead box A1 protein. Oncotarget 7 : $13139-13152,2016$.

36. Luo W, Huang B, Li Z, Li H, Sun L, Zhang Q, Qiu X and Wang E: MicroRNA-449a is downregulated in non-small cell lung cance and inhibits migration and invasion by targeting c-Met. PLoS One 8: e64759, 2013.

37. Wheeler DL, Dunn EF and Harari PM: Understanding resistance to EGFR inhibitors-impact on future treatment strategies. Nat Rev Clin Oncol 7: 493-507, 2010.

38. Sangodkar J, Dhawan NS, Melville H, Singh VJ, Yuan E, Rana H, Izadmehr S, Farrington C, Mazhar S, Katz S, et al: Targeting the FOXO1/KLF6 axis regulates EGFR signaling and treatment response. J Clin Invest 122: 2637-2651, 2012.

39. Chen X, Zhu L, Ma Z, Sun G, Luo X, Li M, Zhai S, Li P and Wang X: Oncogenic miR-9 is a target of erlotinib in NSCLCs. Sci Rep 5: 17031, 2015

40. Li B, Ren S, Li X, Wang Y, Garfield D, Zhou S, Chen X, Su C Chen M, Kuang P, et al: MiR-21 overexpression is associated with acquired resistance of EGFR-TKI in non-small cell lung cancer. Lung Cancer 83: 146-153, 2014.

41. Babu KR and Muckenthaler MU: miR-20a regulates expression of the iron exporter ferroportin in lung cancer. J Mol Med (Berl) 94: 347-359, 2016.

42. Adi Harel S, Bossel Ben-Moshe N, Aylon Y, Bublik DR, Moskovits N, Toperoff G, Azaiza D, Biagoni F, Fuchs G, Wilder S, et al: Reactivation of epigenetically silenced miR-512 and miR-373 sensitizes lung cancer cells to cisplatin and restricts tumor growth. Cell Death Differ 22: 1328-1340, 2015 .

43. Zhao J, Fu W, Liao H, Dai L, Jiang Z, Pan Y, Huang H, Mo Y, Li S, Yang $G$ and Yin J: The regulatory and predictive functions of miR-17 and miR-92 families on cisplatin resistance of non-small cell lung cancer. BMC Cancer 15: 731, 2015.

44. Yamashita R, Sato M, Kakumu T, Hase T, Yogo N, Maruyama E, Sekido Y, Kondo M and Hasegawa Y: Growth inhibitory effects of miR-221 and miR-222 in non-small cell lung cancer cells. Cancer Med 4: 551-564, 2015.

45. Garofalo M, Quintavalle C, Di Leva G, Zanca C, Romano G, Taccioli C, Liu CG, Croce CM and Condorelli G: MicroRNA signatures of TRAIL resistance in human non-small cell lung cancer. Oncogene 27: 3845-3855, 2008.
46. Lv L, An X, Li H and Ma L: Effect of miR-155 knockdown on the reversal of doxorubicin resistance in human lung cancer A549/dox cells. Oncol Lett 11: 1161-1166, 2016.

47. Kalluri R: The biology and function of exosomes in cancer. J Clin Invest 126: 1208-1215, 2016

48. Melo SA, Luecke LB, Kahlert C, Fernandez AF, Gammon ST, Kaye J, LeBleu VS, Mittendorf EA, Weitz J, Rahbari N, et al: Glypican-1 identifies cancer exosomes and detects early pancreatic cancer. Nature 523: 177-182, 2015.

49. Jaiswal R, Gong J, Sambasivam S, Combes V, Mathys JM, Davey R, Grau GE and Bebawy M: Microparticle-associated nucleic acids mediate trait dominance in cancer. FASEB J 26: 420-429, 2012.

50. Pigati L, Yaddanapudi SC, Iyengar R, Kim DJ, Hearn SA, Danforth D, Hastings ML and Duelli DM: Selective release of microRNA species from normal and malignant mammary epithelial cells. PLoS One 5: e13515, 2010.

51. Rabinowits G, Gercel-Taylor C, Day JM, Taylor DD and Kloecker GH: Exosomal microRNA: A diagnostic marker for lung cancer. Clin Lung Cancer 10: 42-46, 2009.

52. Cazzoli R, Buttitta F, Di Nicola M, Malatesta S, Marchetti A, Rom WN and Pass HI: microRNAs derived from circulating exosomes as noninvasive biomarkers for screening and diagnosing lung cancer. J Thorac Oncol 8: 1156-1162, 2013.

53. Rodriguez M, Silva J, López-Alfonso A, López-Muñiz MB, Peña C, Domínguez G, García JM, López-Gónzalez A, Méndez M, Provencio M, et al: Different exosome cargo from plasma/bronchoalveolar lavage in non-small-cell lung cancer. Genes Chromosomes Cancer 53: 713-724, 2014.

54. Zhou X, Wen W, Shan X, Zhu W, Xu J, Guo R, Cheng W, Wang F, Qi LW, Chen Y, et al: A six-microRNA panel in plasma was identified as a potential biomarker for lung adenocarcinoma diagnosis. Oncotarget 8: 6513-6525, 2017.

55. Silva J, García V, Zaballos Á, Provencio M, Lombardía L, Almonacid L, García JM, Domínguez G, Peña C, Diaz R, et al: Vesicle-related microRNAs in plasma of nonsmall cell lung cancer patients and correlation with survival. Eur Respir J 37: 617-623, 2011.

56. Aushev VN, Zborovskaya IB, Laktionov KK, Girard N, Cros MP, Herceg Z and Krutovskikh V: Comparisons of microRNA patterns in plasma before and after tumor removal reveal new biomarkers of lung squamous cell carcinoma. PLoS One 8: e78649, 2013.

57. Liu Q, Yu Z, Yuan S, Xie W, Li C, Hu Z, Xiang Y, Wu N, Wu L, Bai L and Li Y: Circulating exosomal microRNAs as prognostic biomarkers for non-small-cell lung cancer. Oncotarget 8 : 13048-13058, 2017.

58. Barile L and Vassalli G: Exosomes: Therapy delivery tools and biomarkers of diseases. Pharmacol Ther 174: 63-78, 2017.

59. Cao M, Seike M, Soeno C, Mizutani H, Kitamura K, Minegishi Y, Noro R, Yoshimura A, Cai L and Gemma A: MiR-23a regulates TGF- $\beta$-induced epithelial-mesenchymal transition by targeting E-cadherin in lung cancer cells. Int J Oncol 41: 869-875, 2012.

60. Kim J, Kim TY, Lee MS, Mun JY, Ihm C and Kim SA: Exosome cargo reflects TGF- $\beta 1$-mediated epithelial-to-mesenchymal transition (EMT) status in A549 human lung adenocarcinoma cells. Biochem Biophys Res Commun 478: 643-648, 2016.

61. Webber J, Steadman R, Mason MD, Tabi Z and Clayton A: Cancer exosomes trigger fibroblast to myofibroblast differentiation. Cancer Res 70: 9621-9630, 2010.

62. Li J, Yu J, Zhang H, Wang B, Guo H, Bai J, Wang J, Dong Y, Zhao Y and Wang Y: Exosomes-derived MiR-302b suppresses lung cancer cell proliferation and migration via TGF $\beta$ RII inhibition. Cell Physiol Biochem 38: 1715-1726, 2016.

63. van den Boorn JG, Schlee M, Coch C and Hartmann G: SiRNA delivery with exosome nanoparticles. Nat Biotechnol 29: 325-326, 2011

64. Xiao X, Yu S, Li S, Wu J, Ma R, Cao H, Zhu Y and Feng J: Exosomes: Decreased sensitivity of lung cancer A549 cells to cisplatin. PLoS One 9: e89534, 2014

65. Jung JH, Lee MY, Choi DY, Lee JW, You S, Lee KY, Kim J and Kim KP: Phospholipids of tumor extracellular vesicles stratify gefitinib-resistant nonsmall cell lung cancer cells from gefitinib-sensitive cells. Proteomics 15: 824-835, 2015.

66. Alvarez-Erviti L, Seow Y, Yin H, Betts C, Lakhal S and Wood MJ: Delivery of siRNA to the mouse brain by systemic injection of targeted exosomes. Nat Biotechnol 29: 341-345, 2011.

67. Cooper JM, Wiklander PB, Nordin JZ, Al-Shawi R, Wood MJ, Vithlani M, Schapira AH, Simons JP, El-Andaloussi S and Alvarez-Erviti L: Systemic exosomal siRNA delivery reduced alpha-synuclein aggregates in brains of transgenic mice. Mov Disord 29: 1476-1485, 2014 
68. Morse MA, Garst J, Osada T, Khan S, Hobeika A, Clay TM Valente N, Shreeniwas R, Sutton MA, Delcayre A, et al: A phase I study of dexosome immunotherapy in patients with advanced non-small cell lung cancer. J Transl Med 3: 9, 2005.

69. Besse B, Charrier M, Lapierre V, Dansin E, Lantz O, Planchard D, Le Chevalier T, Livartoski A, Barlesi F, Laplanche A, et al: Dendritic cell-derived exosomes as maintenance immunotherapy after first line chemotherapy in NSCLC. OncoImmunology 5: e1071008, 2015.

70. Pitt JM, André F, Amigorena S, Soria JC, Eggermont A, Kroemer G and Zitvogel L: Dendritic cell-derived exosomes for cancer therapy. J Clin Invest 126: 1224-1232, 2016.

71. Marleau AM, Chen CS, Joyce JA and Tullis RH: Exosome removal as a therapeutic adjuvant in cancer. J Transl Med 10: 134, 2012.

72. Chen D, Guo W, Qiu Z, Wang Q, Li Y, Liang L, Liu L, Huang S, Zhao Y and He X: MicroRNA-30d-5p inhibits tumour cell proliferation and motility by directly targeting CCNE2 in non-small cell lung cancer. Cancer Lett 362: 208-217, 2015.

73. Yanaihara N, Caplen N, Bowman E, Seike M, Kumamoto K, Yi M, Stephens RM, Okamoto A, Yokota J, Tanaka T, et al: Unique microRNA molecular profiles in lung cancer diagnosis and prognosis. Cancer Cell 9: 189-198, 2006.
74. Rolfo C, Castiglia M, Hong D, Alessandro R, Mertens I, Baggerman G, Zwaenepoel K, Gil-Bazo I, Passiglia F, Carreca AP, et al: Liquid biopsies in lung cancer: The new ambrosia of researchers. Biochim Biophys Acta 1846: 539-546, 2014.

75. Xu W, Yang Z and Lu N: From pathogenesis to clinical application: Insights into exosomes as transfer vectors in cancer. J Exp Clin Cancer Res 35: 156, 2016.

76. György B, Hung ME, Breakefield XO and Leonard JN: Therapeutic applications of extracellular vesicles: Clinical promise and open questions. Annu Rev Pharmacol Toxicol 55: 439-464, 2015.

77. Kourembanas S: Exosomes: Vehicles of intercellular signaling, biomarkers, and vectors of cell therapy. Annu Rev Physiol 77: 13-27, 2015. 